\title{
Alianzas y redes de colaboración entre las agrupaciones culturales de las Artes Escénicas en Andalucía
}

\author{
Partnerships and collaborative networks between organizations \\ for Performing Arts in Andalusia
}

\author{
Ramos, I. \& Maya-Jariego, I. \\ Universidad de Sevilla \\ isidromj@us.es (ESPAÑA)
}

Recibido: 20.122011

Aceptado: 27.02.2013

\section{RESUMEN}

El análisis de redes sociales se ha utilizado de manera efectiva en la evaluación de programas (Eisenberg \& Swanson, 1996; Fujimoto, Valente \& Pentz, 2009), la gestión de información y la difusión de innovaciones (Valente, 1995; Kraatz, 1998; Ahuja, 2000), el estudio de la evolución de las coaliciones comunitarias (Feinberg, Riggs \& Greenberg, 2005; Wendel et al., 2010) y las iniciativas para promover la capacidad de las organizaciones (Nowel \& Foster-Fishman, 2010). En este estudio se analizan 6 tipos de relaciones entre 32 organizaciones culturales del sector de las artes escénicas en Andalucía. Las agrupaciones de las Artes Escénicas en Andalucía conforman una estructura centro-periferia. El espacio central se articula en torno a una tríada formada por tres entidades jóvenes que destacan, respectivamente, por (a) gestionar recursos de formación en artes escénicas, (b) coordinar la asociación de los profesionales del sector y (c) tener un perfil activo en la producción de espectáculos en los últimos años. A través

${ }^{1}$ Este trabajo forma parte del proyecto «Condiciones laborales, compromiso con la comunidad y redes organizativas en el sector de la industria cultural de Andalucía (CP-0687)». Fundación de Investigación de la Universidad de Sevilla, CGT-0227. Una versión del mismo fue presentada en el «Workshop Internacional de Investigación en análisis de redes sociales», organizado por la Universidad de León y el Campus del Noroeste de la UNED. La versión previa se ha beneficiado de las amables sugerencias de Steve Borgatti, Cristóbal Casanueva y Pilar Marqués. Correspondencia con los autores: Isidro Maya Jariego, Departamento de Psicología Social, Universidad de Sevilla, Campus Ramón y Cajal, Calle Camilo José Cela s/n. 41.018 Sevilla (Spain). E-mail: isidromj@us.es

EMPIRIA. Revista de Metodología de Ciencias Sociales. N. ${ }^{\circ}$ 26, julio-diciembre, 2013, pp. 15-34. ISSN: 1139-5737, DOI: 10.5944/empiria.26.7151 
del análisis de clúster han sido identificados cuatro conglomerados integrados por organizaciones que han desplegado diferentes estrategias relacionales y cuentan con características diferenciales. Los resultados se devolvieron a representantes del sector en un grupo de discusión. Se presentan las reacciones de los participantes.

\title{
PALABRAS CLAVE
}

Redes Inter-organizativas; Estructura Centro-periferia; Visualización; Centralidad; Artes Escénicas.

\begin{abstract}
Social Network Analysis has been used effectively in program evaluation (Eisenberg \& Swanson, 1996; Fujimoto, Valente \& Pentz, 2009), information management and innovations diffusion (Valente, 1995; Kraatz, 1998; Ahuja, 2000), the study of the evolution of community coalitions (Feinberg, Riggs \& Greenberg, 2005; Wendel et al., 2010) and as a tool tool to build organizational capacity (Nowel \& Foster-Fishman, 2010). In this study, we analyzed six types of relationships among 32 cultural organizations in the performing arts sector in Andalusia. This set of organizations of the Performing Arts in Andalusia form a core-periphery structure. The central space is articulated around a triad of three entities characterized, respectively, by (a) managing resources for training, (b) coordinating the professional association and (c) deploying an active role in the production of shows in recent years. Through cluster analysis we identified four subsets. Each cluster is composed by organizations that have displayed different relational strategies and have different characteristics. The results were presented to representatives of organizations in a focus group. Finally we present the participants' reactions.
\end{abstract}

\section{KEYWORDS}

Inter-organizational Networks; Core-periphery Structure; Visualization; Centrality; Performing Arts.

\section{INTRODUCCIÓN}

El análisis de redes se ha aplicado en la evaluación del funcionamiento de las coaliciones comunitarias (Feinberg, Riggs \& Greenberg, 2005; Haines, Godley \& Hawe, 2011), la coordinación de alianzas para la provisión de servicios pú- 
blicos (Provan, Huang \& Milward, 2009; Provan, \& Isset, 2005) y la promoción del desarrollo de innovaciones en contextos empresariales (Danaraj \& Parkhe, 2006; Uzzi \& Spiro, 2005), generando nuevos desarrollos en el estudio de las organizaciones. Todas estas aplicaciones, a pesar de diferir en la finalidad de sus objetivos y en los ámbitos de aplicación, han encontrado en los patrones de relación entre organizaciones respuestas a demandas específicas.

El análisis de redes sociales (ARS) permite identificar las propiedades estructurales de un sistema social determinado (por ejemplo, una coalición interorganizativa), centrando el foco de atención en las relaciones de intercambio que se producen entre las unidades pertenecientes al sistema. De forma operativa, las decisiones sustanciales del proceso de investigación se refieren (a) la adecuada definición del tipo de relación, (b) la delimitación del colectivo objeto de estudio y (c) las características de la vinculación (Knoke \& Yang, 2008, p. 8).

El estudio de las redes inter-organizativas conlleva elementos diferenciales, en comparación con otras unidades de análisis. Los vínculos que se pretenden explorar responden a relaciones que la organización establece con otras entidades. Por tanto resulta de vital importancia identificar a aquellas personas que pueden informar con exactitud de los contactos que su organización establece con otras entidades. En ese sentido, la utilización de informantes clave ha mostrado ser una estrategia efectiva para garantizar un nivel óptimo de adecuación en la descripción de los contactos inter-organizativos (Morrisey, Johnsen \& Calloway, 1997).

La determinación de las relaciones entre organizaciones proporciona una base descriptiva de gran valor. En las páginas que siguen a continuación, desarrollamos un estudio de caso de las redes de colaboración entre las agrupaciones de las artes escénicas en Andalucía. Para ello seguimos un diseño eminentemente inductivo, que parte de las relaciones entre entidades culturales para elaborar una representación del sector. El artículo ilustra el uso de estrategias de carácter descriptivo en la elaboración teórica de las dinámicas de colaboración empresarial. En el siguiente apartado detallamos los objetivos de investigación que han guiado la detección de patrones de interacción específicos en el mundo de la danza.

\section{OBJETIVOS}

El primer objetivo de este estudio consiste en mostrar el núcleo central de relaciones que conforma la red de organizaciones de las artes escénicas en Andalucía. Concretamente, examinamos un subconjunto de entidades que permanecen conectadas a través de diversos tipos de relaciones de intercambio. La multiplicidad de relaciones constituye, de hecho, un indicador de la solidez y la estabilidad de un vínculo entre organizaciones. En la medida en que se extinga una modalidad de relación, aún seguirán otras activas que permitan mantener el vínculo inter-organizativo (Granovetter, 1985; Lazega \& Pattison, 1999).

EMPIRIA. Revista de Metodología de Ciencias Sociales. N. ${ }^{\circ}$ 26, julio-diciembre, 2013, pp. 15-34. ISSN: 1139-5737, DOI: 10.5944/empiria.26.7151 
En segundo lugar, exploraremos las propiedades estructurales de diferentes formas de colaboración, correspondientes a los diferentes tipos de relaciones analizadas. Esta información puede ayudarnos a caracterizar el contexto relacional en el cuál se desenvuelven las organizaciones del sector escénico. La comparación de la estructura de diferentes redes entre un mismo conjunto de actores permite conocer la tipología de relaciones que orienta la formación de alianzas estratégicas en un sector de actividad (Paulson, 1985). Para alcanzar este objetivo evaluaremos la estructura centro-periferia siguiendo el modelo continuo propuesto por Borgatti \& Everett (1999, p. 376) empleando para ello el software Ucinet 6.0 (Borgatti, Everett \& Freeman, 2002). De igual modo analizaremos el grado de superposición entre relaciones a través del procedimiento QAP ( Quadratic Assignment Procedure). Se trata de una técnica que permite calcular las correlaciones entre dos matrices cuadradas, que se usa habitualmente para testar patrones de asociación entre redes (Krackhardt, 1987).

El tercer objetivo es identificar a los actores más prominentes, activos e influyentes en el sector de la danza en Andalucía. Los actores que presentan elevados niveles de centralidad e intermediación pueden acceder a múltiples fuentes de información, adelantarse a las innovaciones o conseguir recursos y financiación que les permiten adquirir ventajas respecto a sus competidores (Schaefer, 2011). La posición de los actores determina en parte su influencia en el sector empresarial (Brass, 1984; Brass \& Burkhardt, 1993; Kilduff \& Tsai, 2003). Pondremos de manifiesto algunos de los factores externos que parecen asociados a las posiciones de poder e influencia en las redes evaluadas.

En cuarto lugar examinamos el papel del contexto socio-económico de la industria cultural en los patrones de relación observados. En un trabajo previo mostramos cómo (a) los elevados niveles de estacionalidad y precariedad laboral, (b) la dependencia económica de la Administración Pública y (c) la particular idiosincrasia de la industria cultural influyen en el modo en que se articulan las relaciones entre las organizaciones del sector escénico andaluz (Santolaya, Ramos Vidal, Jurado, Holgado \& Maya Jariego, 2011). Señalaremos cuáles son los factores que de modo más significativo parecen configurar la estructura de la red inter-organizativa.

Finalmente, agrupamos a las distintas organizaciones en función del patrón de relaciones desplegado. De ese modo, identificamos diferentes tendencias y estilos en el establecimiento de contactos con el resto de organizaciones del sector. En esa sección describiremos las principales características de las organizaciones de cada subgrupo y las diferencias existentes entre ellas. A continuación resumimos las estrategias de investigación desarrolladas en el estudio de caso.

\section{MÉTODO}

Seguimos un diseño inductivo de investigación, en una secuencia que combina (a) el análisis iterativo de indicadores de centralidad y agrupación de las redes inter-organizativas, (b) la representación gráfica de relaciones, (c) la devolución 
y discusión cualitativa con informantes clave y (d) la información descriptiva sobre el contexto socio-económico del sector.

El análisis de datos se desarrolla en cuatro etapas. Primero se describe la estructura de la red y la centralidad de los actores. En segundo lugar, se analiza el grado de asociación entre seis tipos de relación diferentes. A continuación, se identifican las organizaciones más relevantes en el sector. Para profundizar en las propiedades de la red se proporciona información sobre el contexto socioeconómico y se recurre a la interpretación de informantes clave. Finalmente, se clasifican las organizaciones en conglomerados. La sección de resultados se organiza en cuatro apartados, correspondientes a las cuatro etapas del análisis de datos.

\section{Participantes}

El objeto de estudio fueron las 41 agrupaciones de danza existentes en las ocho provincias andaluzas, según el registro del Centro de Documentación de las Artes Escénicas de la Consejería de Cultura de la Junta de Andalucía (Santolaya et al., 2011). En total fueron consultadas 32 organizaciones, que constituyen el 75 por ciento de la población. Para obtener la información sobre las relaciones entre entidades, entrevistamos a un representante de cada organización. En la mayoría de los casos se trataba del director de la entidad, y generalmente mostraban disponer de un conocimiento detallado sobre los contactos externos de la agrupación.

La industria cultural es un sector con entidad propia. Entre otros aspectos, se caracteriza por (a) el carácter estacional de la actividad, (b) la dependencia de la Administración Pública para la obtención de recursos y (c) la naturaleza peculiar del producto (cultural) resultante. En la tabla 1 exponemos algunos datos descriptivos de las organizaciones analizadas.

Tabla 1. Estadísticos descriptivos de las características de las organizaciones evaluadas

\begin{tabular}{lcc}
\hline \multicolumn{1}{c}{ Características } & M & DT \\
\hline Años de actividad & 9,8 & 8,52 \\
Número de integrantes & 7,9 & 18,09 \\
Volumen de facturación $(€)$ & 139.238 & 284.188 \\
Número de eventos anuales & 45 & 67 \\
\hline
\end{tabular}

Fuente: Elaboración propia a partir del informe de investigación de Santolaya, Ramos Vidal, Jurado, Holgado \& Maya Jariego (2011)

Las organizaciones presentan una elevada desviación tipo en todas las dimensiones organizativas examinadas, mostrando cierta heterogeneidad interna 
del sector. Normalmente se trata de empresas pequeñas, compuestas por unos 7 trabajadores en promedio, en las que se difumina la estructura organizativa y apenas se observa una división estructurada del trabajo. Los representantes de las organizaciones tienen una edad promedio de 39 años, y se reparten de forma equilibrada entre hombres (el 51.6\%) y mujeres $(48,4 \%)$. En líneas generales se trata de personal con un elevado nivel de cualificación. En torno al $75 \%$ cuenta con estudios universitarios de segundo ciclo, llevan trabajando 18 años de media en el sector y reciben un sueldo bruto anual de 12.000 euros.

\section{Tipología de vínculos analizados}

El análisis de redes se basó en seis tipos distintos de relaciones. Cada uno de ellos ofrece una red de intercambios sustancialmente diferente, con independencia de que se trate del mismo conjunto de actores (Knoke \& Yang, 2008; Scott, 1991). De hecho, exploramos relaciones que conllevan niveles diferentes de compromiso y acuerdo entre los actores, asumiendo que los intercambios entre organizaciones suelen evolucionar desde la informalidad de los contactos iniciales hasta la normatividad de los vínculos formalmente definidos (Gulati \& Gargiulo, 1999, p. 1440). Concretamente, evaluamos (a) relaciones que no requieren compromiso explícito alguno (el mero reconocimiento de otra entidad del sector), (b) el establecimiento de contactos informales y (c) formales, (d) la percepción de conexión compartida, (e) la posibilidad futura de contactos y (f) la participación conjunta en proyectos. A priori, cada relación requiere un grado de acuerdo progresivo, hasta llegar al desarrollo de proyectos comunes. Este último nivel conlleva un grado considerable de compromiso relacional para llevar a cabo iniciativas empresariales de forma conjunta.

La red de reconocimiento de otras entidades y la participación conjunta en proyectos se evaluaron de forma binaria (1=existencia de relación; $0=$ ausencia). En las otras cuatro redes se valoró la intensidad de los contactos, de forma que los entrevistados debían ponderar (de 0 a 3 ) la fuerza de la relación. A partir de las 6 redes completas, elaboramos una matriz agregada de contactos en la que incorporamos las puntuaciones de cada díada en cada una de las seis relaciones analizadas. Esto permite integrar la multiplicidad de relaciones en una red conjunta de carácter ponderado.

La identificación de los actores prominentes e influyentes se llevó a cabo computando las medidas de centralidad para cada actor en cada una de las redes de intercambio evaluadas. Hemos calculado los valores de la centralidad de grado e intermediación de cada entidad (Freeman, 1979). También nos hemos apoyado en la visualización para conocer el posicionamiento de los actores en las redes analizadas (Brandes \& Kenis, 2005).

Otro elemento destacado del estudio consistió en la realización de un grupo de discusión con representantes de las organizaciones analizadas (Callejo, 2001, Krueger \& Casey, 2000). La dinámica grupal se centró en la devolución de los resultados de las redes (principalmente a través de la visualización) para conocer 
las reacciones de los miembros del sector, siguiendo la metodología propuesta por Maya-Jariego \& Holgado (2005). Los participantes en el grupo de discusión examinaron los factores que pueden incidir en (a) el posicionamiento central de los actores, (b) el aislamiento relacional y (c) las características generales de la red inter-organizativa. En el grupo de discusión participaron informantes clave, con indicadores elevados de centralidad, que suelen tener una percepción más ajustada de la red en su conjunto (Brass, 1984; Krackhardt, 1990, p.345).

Para identificar conglomerados llevamos a cabo un análisis de clúster con el procedimiento de optimización (Borgatti, et al., 2002), tomando como referencia la red global de contactos. El análisis de conglomerados permite asignar casos a un número definido de subgrupos, cuyas características son a priori desconocidas pero están basadas en variables especificadas por los investigadores (Kaufman \& Rousseeuw, 2008).

\section{RESULTADOS}

\section{Descripción de las redes de colaboración en las artes escénicas}

Utilizando la matriz agregada de relaciones, representamos con Netdraw los vínculos fuertes entre organizaciones $(>2)$. El gráfico 1 representa la red global de relaciones. Esta información constituye un indicador de la multiplicidad de contactos, reflejando múltiples contenidos relacionales e, indirectamente, cierto grado de estabilidad en la relación (Granovetter, 1985).

Figura 1. Red agregada de contactos identificando lazos fuertes

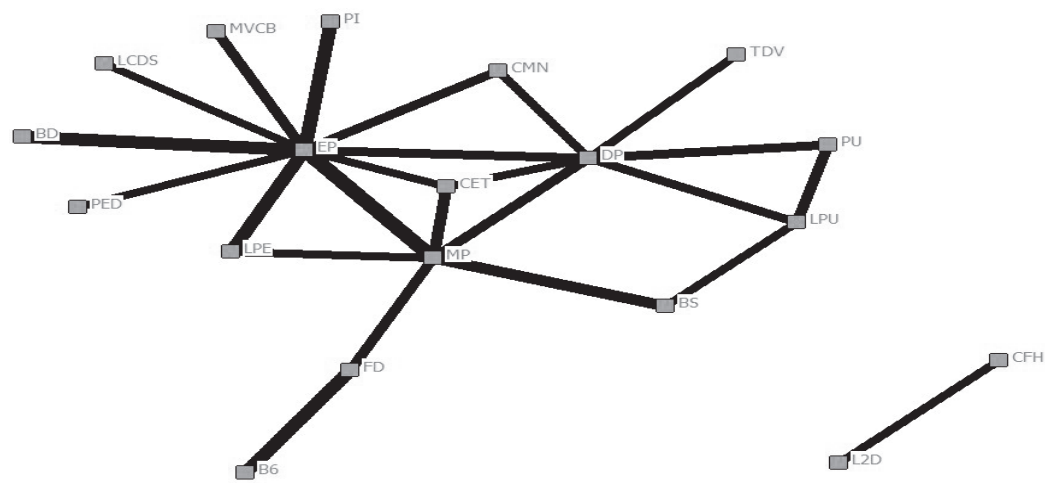

Como podemos observar en la figura 1, el núcleo central de relaciones está conformado por 19 de las 32 organizaciones inicialmente evaluadas. Este subconjunto principal incluye entidades que se encuentran densamente conectadas entre sí a través de múltiples tipos de relaciones caracterizadas por su elevada intensidad. En su mayoría se trata de organizaciones radicadas en la provincia 
de Sevilla: es probable que los costes asociados al establecimiento de una estrategia activa de contactos sean sustancialmente menores que si se tratara de organizaciones distribuidas en otras provincias andaluzas. Este factor facilita la conectividad entre los representantes de las organizaciones del sector, dando lugar a una elevada densidad en las relaciones sin necesidad de incurrir para ello en excesivos costes financieros o temporales.

De hecho, existe un alto de grado de concentración de las iniciativas de las artes escénicas en la provincia de Sevilla. Por ejemplo, (a) los principales centros formativos especializados en artes escénicas, (b) las instituciones de la Administración de interés para el sector cultural y (c) los eventos especializados, tienen lugar en la provincia de Sevilla. Se produce cierta coincidencia entre el alto grado de concentración de organizaciones en la provincia (el 50\% del censo completo) y el alto nivel de conexión entre las organizaciones del subconjunto principal. La concentración de iniciativas en la provincia de Sevilla facilita a las organizaciones estar adecuadamente conectadas con unos costes moderados, mientras que para las organizaciones de otras provincias puede suponer costes mayores, en algunos casos difíciles de asumir. En este contexto, la sucesión de contactos haría posible construir relaciones sólidas en las que las partes habrán gozado de múltiples oportunidades para conocerse mejor y diseñar, en consecuencia, estrategias de colaboración basadas en intercambios satisfactorios previos.

\section{Asociación entre los diferentes tipos de relaciones de colaboración}

En segundo lugar, profundizamos en el conocimiento de las propiedades estructurales de las redes de intercambio analizadas, mostrando el nivel de superposición entre los distintos vínculos. Para ello evaluamos la estructura centroperiferia (Borgatti \& Everett, 1999), comparando ambos conjuntos en atención a dos de las principales medidas de cohesión: la densidad y la reciprocidad. La densidad es una medida que se expresa como porcentaje de las relaciones existentes sobre el total de relaciones posibles, mientras que la reciprocidad indica el número de lazos que son retornados a los emisores, confirmando indirectamente el nivel de exactitud en la formalización de la relación (Wasserman \& Faust, 1994). En la tabla 2 se indican los valores de las medidas de cohesión en las subestructuras central y periférica.

Tabla 2. Valores correspondientes a la densidad y reciprocidad en la estructura centro-periferia

\begin{tabular}{lcccc}
\hline \multicolumn{1}{c}{ Tipo de relación } & $\begin{array}{c}\text { Densidad } \\
\text { centro }\end{array}$ & $\begin{array}{c}\text { Densidad } \\
\text { periferia }\end{array}$ & $\begin{array}{c}\text { Reciprocidad } \\
\text { centro }\end{array}$ & $\begin{array}{c}\text { Reciprocidad } \\
\text { periferia }\end{array}$ \\
\hline Reconocimiento entidad & 0.9476 & 0.2941 & 0.8952 & 0.2698 \\
Contactos Informales & 0.2107 & 0.0121 & 0.1881 & 0.2500
\end{tabular}

EMPIRIA. Revista de Metodología de Ciencias Sociales. N. o 26, julio-diciembre, 2013, pp. 15-34. ISSN: 1139-5737, DOI: 10.5944/empiria.26.7151 


\begin{tabular}{lcccc}
\hline \multicolumn{1}{c}{ Tipo de relación } & $\begin{array}{c}\text { Densidad } \\
\text { centro }\end{array}$ & $\begin{array}{c}\text { Densidad } \\
\text { periferia }\end{array}$ & $\begin{array}{c}\text { Reciprocidad } \\
\text { centro }\end{array}$ & $\begin{array}{c}\text { Reciprocidad } \\
\text { periferia }\end{array}$ \\
\hline Contactos Formales & 0.2500 & 0.0575 & 0.3750 & 0.1765 \\
Percepción de conexión & 0.1351 & 0.0444 & 0.5581 & 0.0000 \\
Contactos futuros & 0.2550 & 0.0010 & 0.3398 & 0.0000 \\
Participación conjunta & 0.5330 & 0.0425 & 0.3472 & 0.3000 \\
\hline
\end{tabular}

Las puntuaciones de la tabla 2 informan de la amplia variabilidad en el nivel de conectividad existente entre el subconjunto de organizaciones que ocupan posiciones centrales y las que forman la constelación externa de la red. Como era de esperar, en el caso de la densidad se observan valores muy superiores en el centro en comparación con la periferia. Especialmente amplia es la variación en esta medida de cohesión en las redes de reconocimiento de otras entidades y de participación conjunta en eventos, que constituyen los vínculos que requieren de menor y mayor nivel de compromiso, respectivamente.

Las organizaciones se distribuyen casi a partes iguales entre el centro y la periferia. Las entidades que ocupan el centro están densamente conectadas entre sí mientras que las organizaciones externas al núcleo central se encuentran débilmente relacionadas. La reciprocidad sigue en líneas generales un patrón similar. Las diferencias más evidentes se dan en el caso de las redes de percepción de conexión y de posibilidad futura de contactos, en las que no existe reciprocidad en la estructura periférica. No obstante, en las redes de participación conjunta en eventos y especialmente en la red de contactos informales hemos observado un patrón sustancialmente diferente caracterizado por niveles similares e incluso superiores de reciprocidad en la estructura periférica en comparación con la central.

Especialmente llamativo es el caso de la participación conjunta en eventos, puesto que supone un mayor nivel de compromiso y por tanto podríamos esperar mayores niveles de reciprocidad en el núcleo de organizaciones más conectado. El mismo fenómeno se observa con los contactos informales. Es probable que para los participantes tengan menos relevancia los contactos informales cuando ya existen otro tipo de contactos inter-organizativos, extremo altamente probable en la estructura central, densamente conectada. En otros términos, lo que parecen mostrar estos datos es que la respuesta a los diferentes tipos de relaciones no son independientes entre sí.

Para conocer el grado en que dos tipos de relaciones tienden a aparecer de forma simultánea, hemos analizado el nivel de superposición entre relaciones a través del procedimiento QAP. Este análisis permite conocer la medida en la que cada díada tiende a establecer diferentes relaciones de forma concurrente, al mismo tiempo que nos ofrece información de carácter global sobre el patrón relacional inter-organizativo.

En la tabla 3 exponemos la información correspondiente a las correlaciones QAP, utilizando como indicadores de superposición el coeficiente de correla- 
ción de Pearson $(r)$ y el coeficiente de Jaccard $(C J)$. De acuerdo con Friedkin (2009), este último indicador constituye un índice que permite analizar el nivel de superposición entre redes. Los valores del mismo, que se interpretan a modo de porcentaje, oscilan entre 0 y 1: cuanto más se aproxime el valor a la unidad indica un mayor grado de superposición entre redes y viceversa.

Los datos muestran un alto grado de solapamiento entre las relaciones exploradas. Dicha superposición oscila entre el $48 \%$ existente entre las redes de contactos formales e informales y el $30 \%$ entre los contactos formales y la participación conjunta en eventos. Las organizaciones tienden a establecer múltiples relaciones entre sí al mismo tiempo.

Tabla 3. Correlaciones diádicas entre matrices a través del procedimiento QAP

\begin{tabular}{lllllll}
\hline & \multicolumn{2}{c}{$\begin{array}{c}\text { Contactos } \\
\text { informales }\end{array}$} & \multicolumn{2}{c}{$\begin{array}{c}\text { Contactos } \\
\text { futuros }\end{array}$} & \multicolumn{2}{c}{$\begin{array}{l}\text { Percepción } \\
\text { de conexión }\end{array}$} \\
\hline & $\mathrm{R}$ & $\mathrm{CJ}$ & $\mathrm{R}$ & $\mathrm{CJ}$ & $\mathrm{R}$ & $\mathrm{CJ}$ \\
\hline Contactos formales & .71 & 0.48 & .38 & 0.30 & .33 & 0.33 \\
Contactos informales & & & .48 & 0.34 & .44 & 0.38 \\
Contactos futuros & & & & & .62 & 0.47 \\
\hline
\end{tabular}

La correspondencia entre la red de contactos formales e informales muestra que las organizaciones establecen contactos de carácter formal con aquellas entidades con las que han establecido contactos informales y viceversa. Los contactos informales permiten a las organizaciones adquirir un conocimiento crítico sobre sus socios potenciales y desarrollar experiencias positivas sobre las que poder construir alianzas estratégicas. Las relaciones informales también proporcionan experiencias que se traducen en términos de estatus o reputación, que ejercen de facilitadores en la formación de coaliciones (Granovetter, 1985; Larson, 1992; Provan et al., 2009).

También se observa un nivel de solapamiento elevado (el 47\%) entre la posibilidad futura de contactos y la percepción de conexión. Parece que las organizaciones tendrán mayor predisposición a participar conjuntamente en proyectos con aquellas entidades con las que perciben que tienen valores comunes. Esta información coincide con el estudio de Zajonc \& Edwards (2006), que determinó que la percepción de similitud en los valores constituye uno de los elementos que predice la formación de coaliciones.

\section{Roles y estrategias de los actores con mayor centralidad}

El tercer paso consistió en identificar a los actores más centrales e influyentes en las distintas redes evaluadas. En este caso utilizamos también la matriz agregada de contactos, puesto que proporciona de un modo más fiable el núcleo central de relaciones en torno a las cuales se estructura la red. La figura 2 repre- 
senta el sociograma completo de relaciones fuertes, señalando con un círculo de puntos a los actores más centrales. El núcleo pivota sobre los cuatro actores con mayor centralidad. Dichas organizaciones presentan valores elevados en la centralidad de grado e intermediación en varios de los seis tipos de relaciones analizados. Aunque no necesariamente han presentado los valores más elevados en cada una de ellas, destacan en su conjunto en los indicadores de centralidad, lo que se traduce en posiciones de influencia en la red global de relaciones.

Figura 2. Red completa de lazos fuertes identificando a los actores centrales

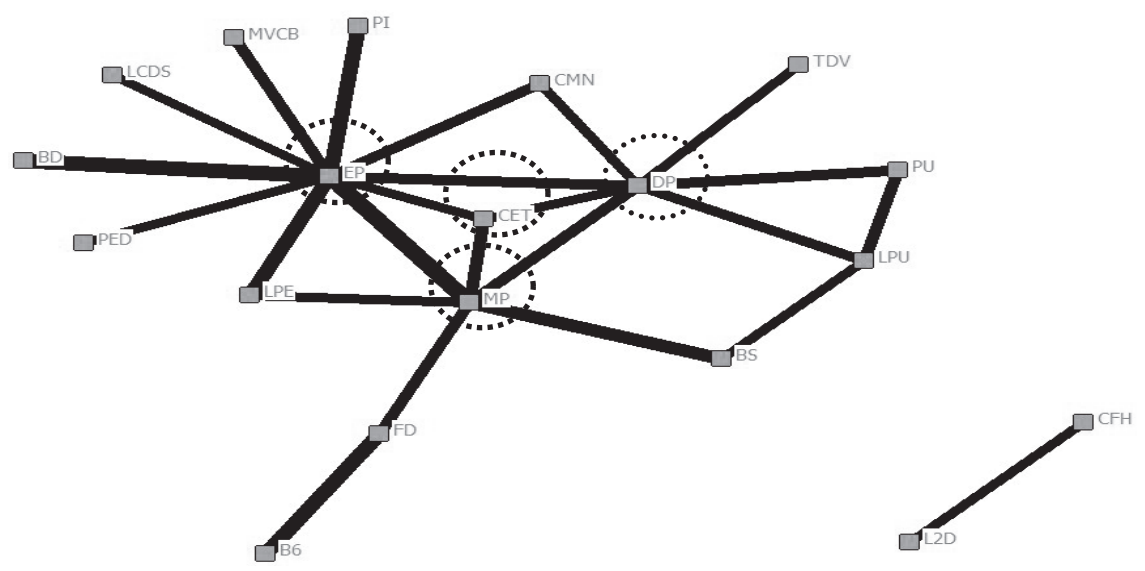

El posicionamiento destacado de determinadas organizaciones se contrastó con información cualitativa. Tanto el análisis de las medidas de centralidad como la visualización de las relaciones pueden combinarse con la interpretación de los actores, aportando facilitadores e inhibidores de las posiciones de poder e influencia (Maya Jariego \& Holgado, 2005). En este caso llevamos a cabo un grupo de discusión con nueve representantes de las organizaciones más centrales, que señalaron factores específicos que a su juicio habían permitido a las organizaciones alcanzar posiciones influyentes en las redes examinadas. Están resumidos en la Tabla 4.

Uno de los factores que incrementa la conectividad de las organizaciones se relaciona con el hecho de que la entidad disponga de una persona encargada de las relaciones públicas. Según los participantes, el ejercicio de dicho rol permite establecer una estrategia activa de contactos (a) con representantes de otras organizaciones, (b) con programadores culturales (que son los agentes encargados de seleccionar los espectáculos que se representarán en municipios y en salas independientes) y (c) con la Administración Pública.

Experimentar un período de especial productividad y creatividad artística también contribuye positivamente al posicionamiento central de las organizaciones. El éxito en la producción artística permite a las organizaciones conformar 
una reputación efectiva. Esto incita a otras entidades a establecer alianzas con las organizaciones exitosas para beneficiarse de dicha notoriedad (Larson, 1992).

En tercer lugar, mencionaron el ejercicio de funciones de representación institucional en asociaciones profesionales de defensa de los intereses del colectivo. El desempeño de dichas actividades implica generalmente actuar como interlocutor entre el sector y las instituciones públicas y privadas de referencia. A menudo la función de representación requiere difundir información de interés para el sector (por ejemplo, subvenciones o convocatorias), participar en paneles de expertos o mesas de trabajo y acudir a eventos especializados. Todas estas actividades exigen un alto grado de conexión con diferentes agentes del sector, y facilitan el posicionamiento central y la visibilidad de las organizaciones cuyos miembros desempeñan este tipo de rol.

Tabla 4. Factores facilitadores de un posicionamiento central

\begin{tabular}{|c|c|}
\hline Organización & Factores que facilitan la centralidad y la influencia \\
\hline E.P & $\begin{array}{l}\text { - Cuenta con una persona dedicada en exclusiva a las RRPP. } \\
\text { - La organización conduce uno de los principales centros de } \\
\text { formación en artes escénicas en Andalucía. }\end{array}$ \\
\hline D.P & $\begin{array}{l}\text { - Cuenta con una persona encargada de la distribución-produc- } \\
\text { ción. } \\
\text { - La directora es representante de la PAD. }\end{array}$ \\
\hline M.P & $\begin{array}{l}\text { - Organización exitosa en la creación artística. } \\
\text { - Ha formado coaliciones con entidades externas a la red. } \\
\text { - Diversidad/heterogeneidad que conduce a mayores niveles de } \\
\text { creatividad (Uzzi \& Spiro, 2005). } \\
\text { - Es observada como socio potencial. }\end{array}$ \\
\hline C.E.T & $\begin{array}{l}\text { - Dilatada trayectoria en el sector. } \\
\text { - Persona encargada de distribución-producción. } \\
\text { - Dispone de un espacio escénico que pone al servicio de otras } \\
\text { entidades. }\end{array}$ \\
\hline
\end{tabular}

\section{Análisis de conglomerados de las organizaciones de artes escénicas}

En esta sección analizamos los factores que modulan la estructura de la red de organizaciones de las artes escénicas en Andalucía. Para ello nos centramos en la red de participación conjunta en proyectos, porque refleja el nivel más elevado de compromiso inter-organizativo y supone la consecución del nivel de colaboración más avanzado. En la figura 3 se representa el grafo, del cual hemos eliminado a los actores con mayor centralidad de grado, con el objetivo de discernir con mayor claridad los principales subconjuntos en los que puede fragmentarse la red. 
Como se observa en el gráfico 3, distinguimos tres subgrupos en los que intuitivamente puede descomponerse la red. El grupo identificado con un círculo de líneas y puntos discontinuo, está formado por organizaciones radicadas fuera de Sevilla. Se trata sobre todo de organizaciones ubicadas en Andalucía Oriental, que tienen más oportunidades de coincidir en eventos organizados en esa área geográfica, evitando los costes asociados al desplazamiento.

Figura 3. Red de participación conjunta sin actores centrales diferenciando subconjuntos principales

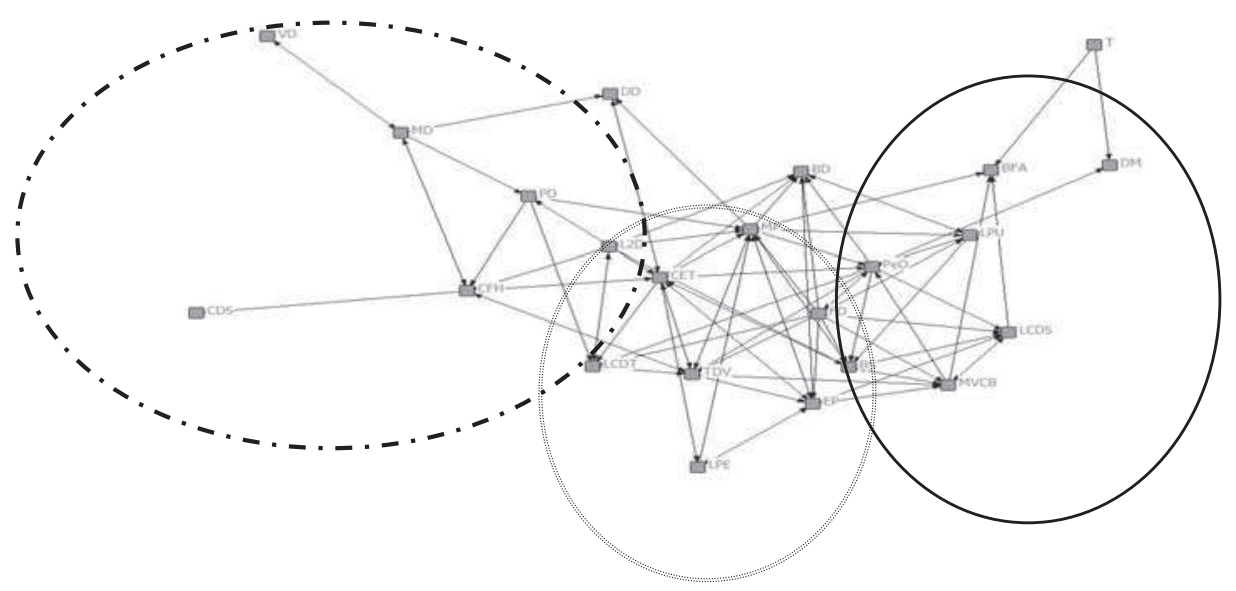

El círculo de puntos delimita un subconjunto amplio de organizaciones que cubren diferentes disciplinas escénicas, principalmente danza y teatro. La hibridación disciplinar les permite acceder a entornos organizativos más diversos y mantener relaciones con instituciones de otros ámbitos. Esta heterogeneidad facilita además el acceso a nuevos recursos y fuentes de información. Estudios previos han apuntado la influencia que la heterogeneidad en la composición de las redes sociales puede producir en la generación de innovaciones creativas (Uzzi \& Spiro, 2005). Estos datos parecen indicar que la diversidad del entramado social en el que se desenvuelven las organizaciones, enriquece sustancialmente la información de la que disponen los miembros de la red para poner en funcionamiento iniciativas creativas.

Finalmente, el círculo de línea continua engloba a las organizaciones especializadas en un área concreta de las artes escénicas. En este caso nos encontramos con el supuesto inverso al anteriormente descrito: se trata de entidades que se han especializado en alguna disciplina de la danza, principalmente el flamenco. Esta distinción tiene de facto importantes implicaciones prácticas en el desarrollo de alianzas entre las organizaciones del sector. El discurso político defiende el tratamiento general y equiparable de las agrupaciones del sector escénico andaluz. Sin embargo, en la práctica existen diferentes instituciones 
encargadas de definir las ayudas al sector y los circuitos en los que participan las agrupaciones dependiendo de su especialización artística. De tal forma que instituciones de reciente creación como la Agencia Andaluza del Flamenco, son las encargadas de administrar las ayudas a las organizaciones dedicadas a este colectivo. Algo similar sucede en el caso de la danza clásica y contemporánea. Este aspecto implica que las organizaciones que se dedican a la misma disciplina, ante cualquier eventualidad administrativa acudirán al mismo organismo, competirán en las mismas convocatorias de ayudas y participarán en los mismos circuitos. Estas múltiples coincidencias pueden favorecer el establecimiento de relaciones sólidas, así como el intercambio progresivo de información y recursos que facilite la construcción de relaciones estables.

El quinto y último objetivo del estudio de caso consistió en establecer una clasificación de las organizaciones, en función de las estrategias relacionales implementadas. Para lograr este propósito llevamos a cabo un análisis de clúster por el procedimiento de optimización. Este modelo de análisis realiza agrupaciones y permite analizar las características compartidas dentro de cada grupo en una matriz de proximidad (Glover, 1989, 1990).

El modelo de optimización con mejor ajuste arrojó cuatro conglomerados de distinto tamaño, con un ajuste final de 0.52 . El primer conglomerado cuenta con cuatro organizaciones (12,5\% del total), el segundo está integrado por 19 entidades $(59,4 \%)$, el tercero por $5(15,6 \%)$ y el último por $4(12,5 \%)$.

Tabla 5. Descripción de las características organizativas por conglomerado

\begin{tabular}{|c|c|c|}
\hline Conglomerado & $\begin{array}{c}\text { N. }{ }^{\circ} \text { y }(\%) \\
\text { de entidades }\end{array}$ & Características de las organizaciones \\
\hline Clúster 1 & $4(12,5 \%)$ & $\begin{array}{l}\text { - Especialización en flamenco. } \\
\text { - Vínculos institucionales. } \\
\text { - Orientación al mercado externo. } \\
\text { - Nivel medio-bajo de conexiones. }\end{array}$ \\
\hline Clúster 2 & $19(59,4 \%)$ & $\begin{array}{l}\text { - Entidades radicadas en la provincia de Sevilla. } \\
\text { - Carácter independiente. } \\
\text { - Especialización en danza contemporánea. } \\
\text { - Nivel elevado de conectividad. }\end{array}$ \\
\hline Clúster 3 & $5(15,6 \%)$ & $\begin{array}{l}\text { - Organizaciones asentadas fuera de Sevilla. } \\
\text { - Trayectoria dilatada en el sector. } \\
\text { - Especialización en nuevas tendencias creativas. } \\
\text { - Conectividad moderada. }\end{array}$ \\
\hline Clúster 4 & $4(12,5 \%)$ & $\begin{array}{l}\text { - Organizaciones asentadas fuera de Sevilla. } \\
\text { - Reciente creación y baja conectividad. } \\
\text { - Dedicación a disciplinas poco extendidas (por } \\
\text { ejemplo, danza oriental). } \\
\text { - Dificultad de participación en circuitos públicos. }\end{array}$ \\
\hline
\end{tabular}

EMPIRIA. Revista de Metodología de Ciencias Sociales. N. ${ }^{\circ}$ 26, julio-diciembre, 2013, pp. 15-34. ISSN: 1139-5737, DOI: 10.5944/empiria.26.7151 
El segundo conglomerado se corresponde con el núcleo cohesivo que identificamos en la figura 1, mientras que los otros tres conglomerados presentan patrones relacionales diferentes en función de sus atributos. Hemos empleado la densidad interna de cada conglomerado como criterio complementario para caracterizar a cada clúster. En la tabla 5 resumimos las características de las organizaciones de cada conglomerado y los factores que afectan a la formación de grupos.

El primer conglomerado incluye a las organizaciones dedicadas al flamenco. Se trata de una parcela de actividad con notas distintivas, y los recursos de este subsector se canalizan a través de instituciones específicas. El segundo conglomerado, el más numeroso, está formado por organizaciones orientadas a la disciplina contemporánea, radicadas en la provincia de Sevilla. Este clúster se caracteriza por presentar los niveles más elevados de conectividad. En sentido contrario los conglomerados 3 y 4 están integrados por entidades externas a la provincia de Sevilla. Los clústeres obtenidos coinciden, por tanto, en líneas generales con las agrupaciones identificadas con las estrategias de visualización exploratoria y con las interpretaciones cualitativas de los participantes.

En resumen, hemos identificado determinados factores como (a) la distribución geográfica, (b) la especialización artística, (c) la hibridación disciplinar o (d) la vinculación institucional, que parecen influir en la conformación de las redes inter-organizativas en el sector de la danza en Andalucía. Estos datos indican que la formación de subconjuntos cohesivos, que dan lugar en muchos casos a alianzas estratégicas, no es fruto del azar. Por el contrario, en la mayoría de los casos depende de factores endógenos atribuibles a las organizaciones (por ejemplo, las características organizativas, la especialidad artística y la orientación de mercado); y factores exógenos imputables al contexto específico del sector (tales como la distribución de recursos, o la concentración de iniciativas e instituciones) que influyen indirectamente en la formación de coaliciones interorganizativas.

\section{CONCLUSIONES}

La red de organizaciones de las artes escénicas en Andalucía está formada por un núcleo central constituido por 19 entidades. Todas las organizaciones que integran este eje se encuentran radicadas en Sevilla. Este fenómeno puede deberse en parte al alto grado de concentración de las instituciones relacionadas con el sector, los centros de formación especializados y los eventos específicos que tienen lugar en la capital hispalense. El caso estudiado muestra la influencia del contexto institucional en la estructuración de la red.

Dentro de ese núcleo central hemos identificado 4 organizaciones que ocupan posiciones de poder e influencia en los diferentes tipos de relaciones evaluadas. El ejercicio de un rol prominente se ve favorecido al disponer de una persona dedicada a visibilizar la imagen de la entidad; desarrollar funciones de representación institucional o estar experimentando un momento álgido en la

EMPIRIA. Revista de Metodología de Ciencias Sociales. N. ${ }^{\circ}$ 26, julio-diciembre, 2013, pp. 15-34. ISSN: 1139-5737, DOI: 10.5944/empiria.26.7151 
productividad artístico-creativa. En sentido inverso, factores tales como la orientación al mercado exterior, la dedicación a disciplinas artísticas poco extendidas en Andalucía e incluso el aislamiento geográfico parecen afectar negativamente al nivel de centralidad de los actores.

Las organizaciones construyen sus alianzas sobre diferentes tipos de relaciones. El análisis de la superposición entre matrices nos ha permitido identificar vínculos que tienden a darse de manera conjunta. Por ejemplo, las agrupaciones deciden poner en marcha iniciativas o proyectos conjuntos una vez que cuentan con antecedentes de intercambios positivos. Por tanto, la reputación social o la existencia de relaciones previas satisfactorias pueden anticipar el establecimiento de alianzas estratégicas.

Por su parte, la localización geográfica, la hibridación disciplinar o la especialización artística, facilitan el desarrollo de alianzas específicas. El contexto institucional, descrito en este caso con las interpretaciones cualitativas de los informantes, parece determinante de las dinámicas de la red inter-organizativa analizada. Las conclusiones más significativas obtenidas en el estudio de caso están resumidas en la tabla 6.

Tabla 6. El caso de la red de agrupaciones de danza en Andalucía

- La red inter-organizativa conforma una estructura centro-periferia.

- El núcleo mejor conectado corresponde a las agrupaciones de danza contemporánea radicadas en Sevilla, que están bien vinculadas con la Administración Pública.

- Los roles de mayor centralidad se corresponden con entidades que realizan un papel institucional o de mediación, ya sea coordinar la asociación de los profesionales de las artes escénicas, disponer de un local de ensayo que utilizan otras entidades o proporcionar formación especializada.

- También facilita el desarrollo de relaciones disponer de un responsable de las relaciones públicas o contar con un volumen elevado de producción cultural reciente.

- Las agrupaciones especializadas en flamenco y las organizaciones de Andalucía oriental conforman conglomerados destacados en la periferia del sector.

- Los contactos informales efectivos suelen ser un antecedente de la formación de alianzas estratégicas de carácter formal.

- Del mismo modo, compartir valores con otras entidades aumenta la probabilidad percibida de relaciones en el futuro inmediato.

A lo largo del estudio de caso de la red de la danza hemos desgranado factores de carácter endógeno y exógeno, que contribuyen a su comprensión. De ahí que para evaluar cualquier estrategia puesta en marcha por las propias organizaciones, para construir o formar parte de alianzas con las que mejorar su posicionamiento estratégico, debe tomar en consideración la naturaleza dual de este fenómeno. Del mismo modo, la detección de factores clave puede utilizarse para fomentar la cooperación entre organizaciones, facilitar la distribución de 
recursos en el sector, promover la colaboración intra e inter-sectorial, y organizar adecuadamente las competencias administrativas con las que ofrecer un servicio efectivo a los agentes del sector.

Desde el punto de vista metodológico, este estudio combina dos innovaciones en el estudio de las redes inter-organizativas que pueden dar lugar a desarrollos posteriores. Primero, la evaluación de la multiplicidad de contactos, combinando matrices de relaciones mutuamente dependientes, puede ayudar a comprender cómo evolucionan los contenidos relaciones de las coaliciones organizativas. En segundo lugar, la interpretación de la visualización de la red por parte de los informantes clave permite ubicar las relaciones en un contexto institucional específico. La utilización de ambas estrategias en un diseño inductivo ilustra cómo la descripción empírica en profundidad proporciona elementos para la elaboración teórica de las dinámicas de redes entre organizaciones.

\section{BIBLIOGRAFÍA}

AHUJA, G. (2000). Collaboration networks, structural holes and innovation: A longitudinal study. Administrative Science Quarterly, 45, 425-455.

BORGATTI, S. P.; EVERETT, M. G. \& FREEMAN, L. (2002). UCINET VI Version 1.0. Natick, MA: Analytic Technologies.

BORGATTI, S. P. \& EVERETT, M. G. (1999). Models of Core/Periphery structures. Social Networks 21, 375-395.

BRANDES, U. \& Kenis, P. (2005). La explicación a través de la visualización de redes. REDES-Revista Hispana para el Análisis de Redes Sociales, 9 (6), 1-19.

BRASS, D. J. (1984). Being in the right place: A structural analysis of individual influence in an organization. Administrative Science Quarterly, 29, 518-539.

BRASS, D. J. \& BURKHARDT, M. E. (1993). Potential power and power use: An investigation of structure and behaviour. Academy of Management Journal, 36, 441470.

CALLEJO, J. (2001). El grupo de discusión: introducción a una práctica de investigación. Barcelona. Ariel.

DANARAJ, C. \& PARKHE, A. (2006). Orchestrating innovation networks. Academy of Management Review, 31 (3), 659-669.

EISENBERG, M. \& SWANSON, N. (1996). Organizational network analysis as a tool for program evaluation. Evaluation and the Health Professions, 19, 488-507.

FEINBERG, M. E.; RIGGS, N. R. \& GREENBERG, M. T. (2005). Social networks and community prevention coalitions. Journal of Primary Prevention, 26 (4), 279-298.

FREEMAN, L. C. (1979). Centrality in Social Networks: Conceptual Clarification. Social Networks, 1, 215-239.

FRIEDKIN, N. E. (2009). Jaccard-Spline index of structural proximity in contact networks. Social Networks, 31(1), 76-84.

FUJIMOTO, K.; VALENTE, T. \& PENTZ, M. A. (2009). Network structural influences on the adoption of evidence-based prevention in communities. Journal of Community Psychology, 37 (7), 830-845.

GLOVER, F. (1989). Tabu Search - Part I. ORSA. Journal on Computing, 1, 190-206.

GLOVER, F. (1990). Tabu Search - Part II. ORSA. Journal on Computing, 2, 4-32.

EMPIRIA. Revista de Metodología de Ciencias Sociales. N. ${ }^{\circ}$ 26, julio-diciembre, 2013, pp. 15-34. ISSN: 1139-5737, DOI: 10.5944/empiria.26.7151 
GRANOVETTER, M. (1985). Economic Action and Social Structure: A Theory of Embeddedness. American Journal of Sociology, 91, 481-510.

GULATI, R. \& GARGIULO, M. (1999). Where Do Interorganizational Networks Come From? The American Journal of Sociology, 104 (5), 1439-1493.

HAINES, V. A.; GODLEY, J. \& HAWE, P. (2011). Understanding Interdisciplinary Collaborations as Social Networks. American Journal of Community Psychology, 47, 1-11.

KAUFMAN, L. \& ROUSSEEUW, P. J. (2008). Introduction, in Finding Groups in Data: An Introduction to Cluster Analysis, John Wiley \& Sons, Inc., Hoboken, NJ, USA.

KILDUFF, M. \& TSAI, W. (2003). Social networks and organizations. Thousand Oaks, CA: Sage.

KNOKE, D. \& YANG, S. (2008). Social Network Analysis. (2nd Ed.). Sage Publications Thousand Oacks. California. C. A.

KRAATZ, M. (1998). Learning by association? Interorganizational networks and adaptation to environmental changes. Academy of Management Journal, 41(6), 621-643.

KRACKHARDT, D. (1987). QAP partialling as a test of spuriousness*1. Social Networks, 9(2), 171-186.

KRACKHARDT, D. (1990). Assessing the political landscape: Structure, cognition and power in organizations. Administrative Science Quarterly, 35, 342-369.

KRUEGER, R. A. \& CASEY, M. A. (2000). (Third edition) Focus groups: A practical guide for applied research. Thousand Oaks, CA: Sage.

LARSON A. (1992). Network dyads in entrepreneurial settings: a study of the governance of exchange relationships. Administrative Science Quarterly, 37(1), 76-104.

LAZEGA, E. \& PATTISON, P. E. (1999). Multiplexity, generalized exchange and cooperation in organizations: a case study. Social Networks, 21, 67-90.

MAYA-JARIEGO, I. \& HOLGADO, D. (2005). Lazos fuertes y proveedores múltiples de apoyo: comparación de dos formas de representación gráfica de las redes personales. Empiria. Revista de metodología de ciencias sociales, 10, 107-127.

MORRISEY, J. P.; JOHNSEN, M. C. \& CALLOWAY, M. O. (1997). Evaluating Performance and Change in Mental Health Systems Serving Children and Youth: An Interorganizational Network Approach. The Journal of Mental Health Administration, 24 (1), 4-22.

NOWEL, B. \& FOSTER-FISCHMAN, P. (2010). Examining Multi-Sector Community Collaboratives as Vehicles for Building Organizational Capacity. American Journal of Community Psychology, 46(3-4).

PAULSON, S. K. (1985). A paradigm for the analysis of interorganizational networks. Social Networks, 7(2), 105-126.

PROVAN, K. G.; HUANG, K. \& MILWARD, H. B. (2009). The Evolution of Structural Embeddedness and Organizational Social Outcomes in a Centrally Governed Health and Human Services Network, Journal of Public Administration Research and Theory, 17, 873-893.

PROVAN, K. G. \& ISSET, K. (2005). The Evolution of Dyadic Interorganizational Relationships in a Network of Publicly Funded Nonprofit Agencies, Journal of Public Administration Research and Theory, 15, 149-165.

SANTOLAYA, F. J., RAMOS VIDAL, I., JURADO, M. J., HOLGADO, D. \& MAYA JARIEGO, I. (2011). Condiciones de trabajo en las industrias culturales andaluzas: redes organizativas y compromiso con la comunidad de los trabajadores de las artes escénicas. Fundación de Estudios Sindicales CCOO-Andalucía. Consejería de Cultura, Junta de Andalucía. 
SCHAEFER, D. R. (2011). Resource characteristics in social exchange networks: Implications for positional advantage. Social Networks, 33(2), 143-151.

SCOTT, J. (1991). Social Network Analysis: a Handbook. London: Sage.

UZZI, B. \& SPIRO, J. (2005). Collaboration and Creativity: The Small World Problem. American Journal of Sociology, 111(2), 447-504.

VALENTE, T. (1995). Network models of the diffusion of innovations. Cresskill N.J: Hampton Press.

WASSERMAN, S. \& FAUST, K. (1994). Social network analysis: Methods and applications. New York. Cambridge University Press.

WENDEL, M. L.; PROCHASKA, J. D.; CLARK, H. R.; SACKETT, S. \& PERKINS, K. (2010). Interorganizational Network Changes Among Health Organizations in the Brazos Valley, Texas. Journal of Primary Prevent, 31 (1-2), 59-68. 